\title{
Evaluation of clinical biochemical laboratory analytical process performance using sigma metrics and quality goal index
}

\author{
Rogious Mbasani ${ }^{1}$, Luo Xigang ${ }^{1 *}$, Nelson Musilanga ${ }^{2}$ \\ 1 Department of Clinical Laboratory Diagnosis, the Third Affiliated Hospital of Jinzhou Medical University, Jinzhou, China \\ 2 Department of Internal Medicine, the First Affiliated Hospital of Jinzhou Medical University, Jinzhou, China \\ *Corresponding Author: Dr. Luo Xigang, Department of Clinical Laboratory Diagnosis, The Third Affiliated Hospital of Jinzhou \\ Medical University, Jinzhou, China. Phone No.: +86 15084155483, Email: luoxigang_0@126.com
}

Received: November 08, 2021; Accepted: December 02, 2021

\begin{abstract}
Background: Application of sigma matrics across clinical laboratory diagnostic process enables identification of errors in methods together with quality goal index can help point out the cause of unacceptable process performance. We believe the analytical performance of the clinical biochemistry laboratory standards do not meet the required quality according to six sigma standards. Aim and Objective: The study aimed at performance evaluation of clinical biochemical laboratory analytical process using sigma metrics and quality goal index. Methods: Sigma $(\sigma)$ for the 20 analytes performed on HITACHI 7600 - 110 was calculated based on absolute bias, total allowable error (TEa) and coefficient of variation (CV). Two level internal quality control data obtained retrospectively from December 2020 to May 2021 was used to calculate the coefficient of variation. Bias was obtained from six month external quality control data. Total allowable error was obtained from three different sources. Quality goal index (QGI) was calculated to identify the cause of unwanted analyte performance. Results: Different sigma scores were obtained for analytes on different total allowable error standard, only triglyceride showed world class performance along all the three total allowable error standards. However, creatine kinase \& total bilirubin showed world class on clinical laboratory improvement amendment (CLIA) and biological variable database (BVD). On the other hand, glucose, and aspertate amino transferase had poor or unacceptable performance on all the total allowable error standards. Conclusion: Six sigma is an excellent tool in evaluating the quality performance of clinical biochemistry laboratory analytical process, quality goal index can help identify the problem and westgard rules can help design individual quality improvement strategy.
\end{abstract}

Keywords: Quality control, Quality improvement, Six sigma, Standards.

\section{INTRODUCTION}

Clinical laboratories are very strong pillars in any health care systems as the results from the laboratory helps other health care providers especially physicians in deciding on the next step for proper patient management. Laboratory results influences more than $70 \%$ of medical decision making in disease diagnosis and patient management. Reliable, timely and accurate results leads to a rightful physician decision which in turn save the patients from unnecessary procedures, treatment and other financial expenses ${ }^{[1,2]}$. For a laboratory to maximize its utility potential, there must be a quality control system in place to check all the stages of laboratory work processes; preanalytical, analytical and post-analytical for reliable, accurate and timely results ${ }^{[2]}$. Therefore, every clinical laboratory must have a system in place to detect and minimize testing errors for continuous improvement.

Clinical laboratories without good quality management system must be aware of many errors which are likely to occur and go undetected [3,4]. Internal quality control (IQC) and external quality assurance schemes (EQAS) are the common quality control schemes for laboratory analytical processes ${ }^{[5]}$. However, the Clinical and Laboratory Standards guideline call for statistical quality control (SQC) for laboratory risk assessments ${ }^{[6,7]}$. Given the potential sources of imprecision and inaccuracy in a clinical biochemistry laboratory, SQC implementation is paramount to monitor the performance of an analyte procedure to detect any relative change from the baseline analyte performance ${ }^{[8,9]}$. The presence of an in-house well designed quality control (QC) strategy can easily signal the laboratory personal of performance deviations from expected and the possible causes can be easily identified. 
Six Sigma metric, is a generally-accepted and proven quality management tool that quantifies the performance of processes as a rate of Defects-Per-Million Opportunities, (DPM, or DPMO). Six Sigma also includes techniques like Define-MeasureAnalyze Improve-Control (DMAIC), together with quality goal index, Root Cause analysis (RCA) can be performed to identify and eliminate defects and/or variations in a clinical biochemistry laboratory analytical process through measures of bias (EQAS) measure of inaccuracy, imprecision (CV\% of IQC) and standard method specific total allowable error ${ }^{[8,10]}$. All laboratory errors in the analytical phase cannot be assessed, detected and eliminated by only running internal and external QCs hence the need for quantification at a scale of 0 to 6 by Sigma metrics [11].

We believe the analytical performance of the clinical biochemistry laboratory standards do not meet the required quality according to six sigma standards.

The current study evaluated the individual performance of twenty (20) biochemical analytes on HITACHI 7600 - 110, automatic biochemistry analyzer at sigma matrics scale. In addition, we assessed for the appropriate quality control (QC) strategy in accordance with Westgard guidelines for appropriate application of Westgard rules. Further calculated quality goal index (QGI) for root cause analysis (RCA) on parameters with $\sigma<4$ to identify the possible cause of non conformity.

\section{MATERIALS AND METHODS}

\section{Materials}

The internal quality control (IQC) data for determining coefficient of variation percentage ( $\mathrm{CV} \%$ ) for the current study was extracted from HITACHI 7600 - 110 automatic biochemistry analyzer between December 1, 2020 and May 31, 2021, at the hospital clinical biochemistry laboratory. HITACHI 7600 - 110 , analyzer is a modular system consisting of; dispensing module (D1), pippeting module (P1) and a solo ion-selective electrode (ISE) module. A total of 20 analytes were tested evaluated, total protein (TP), albumin (ALB), alanine aminotransferase (ALT), aspartate aminotransferase (AST), Y-glutamyl transpeptidase (GGT), alkaline phosphotase (ALP), uric acid (UA), glucose (GLU), triglyceride (TG), total cholesterol (TC), High density lipoprotein (HDL), urea were analyzed performed on D1 module, total bilirubin (TBIL), creatinine (CRE), magnesium (Mg), phosphorus $(\mathrm{P})$, creatine kinase $(\mathrm{CK})$, were performed on $\mathrm{P} 1$ module where sodium (Na) and potassium (K) on ISE module. The IQC material level 2 (LOT: 695, for normal concentration) and level 3 (LOT: 696, for high (abnormal) concentration) for the 18 analytes and (LOT: P1102) for $\mathrm{Na}$ and $\mathrm{K}$ were purchased from Bio-Rad Laboratories Inc. whereas calibrators were got from Randox laboratories.

EQA samples whose data was used to calculate bias $\%$ were supplied by Bio-Rad Laboratories Inc (LOT: 18892604 and 58605725) External quality control was performed monthly in the biochemical laboratory routine quality control project. Thus, the cumulative absolute bias data from January 2020 to March 2021 were used to calculate the average value for each of the 20 analytes.

\section{Statistical analysis}

\section{Bias calculation}

Bias is an indicator of systematic difference between the obtained results by testing laboratory from the expected results mean of the peer group for a test method expressed as a percentage. Bias percentage for each analyte was calculated from Bio -Rad, EQAs as follows;

$$
\text { Bias } \%=\frac{\text { our lab EQAs result }- \text { Peer group mean }}{\text { Peer group mean }} 100
$$

\section{Coefficient of variation (CV)}

Coefficient of variation is a measure of assay variability expressed in percentage of standard deviation (SD) and mean over a period of time. Precision, (CV) for the 20 analytes was calculated for 6 months for two-level quality controls materials obtained from Bio - Rad as follows;

$$
\mathrm{CV}=(\mathrm{SD} / \mathrm{mean}) \times 100
$$

\section{Sigma calculation}

Three total allowable error (TEa) requirements, USA Clinical Laboratory Improvement Amendments of 1988-CLIA '88 (TEacLIA), Biological Variation Database (TEaвvd) specifications and People's Republic of China Health Industry StandardWS/T 403-2012 (TEaws/T), were selected to calculate $\sigma$ for each assay, using the equation Sigma metrics $(\sigma)=($ TEa $\%-\mid$ Bias $\%$ |) / CV\% (for percentage) and $\sigma=($ TEa - |Bias $\mid$ ) / SD (for concentration value). Excel 2019 software (Microsoft Corporation Redmond, Washington State, US) was used for data analysis and tabulation.

\section{Quality Goal Index (QGI)}

The QGI indicator was calculated according to the standard equation below, the indicator was applied in root cause analysis (RCA) to identify the main reasons for non conformity in the test performance of assays with $\sigma<4$ which help to select the best quality improvement strategy. When $\mathrm{QGI}>1.2$, accuracy improvement is prioritized, $0.8 \leq \mathrm{QGI} \leq 1.2$ both accuracy and precision are of concerned and $\mathrm{QGl}<0.8$, precision of the analyte should be prioritized.

$$
\mathrm{QGI}=\operatorname{Bias}(1.5 \times \mathrm{CV} \%) .
$$

\section{Standardized QC sigma charts}

The standardized QC sigma charts were constructed for both levels 1 and level 2 quality control materials as well as for D1 and $\mathrm{P} 1$ modules of the HITACHI 7600 - 110 biochemistry analyzer. The construction of standardized QC sigma charts progressed after registering a CLInet account in CLInet (http://www.clinet.com.cn) and filling in the required parameters such as TEa, bias, and CV in the Six Sigma management menu page ${ }^{[12]}$. This approach allows for a comprehensive review of the performance of all the analytes in a single graph at different levels of the control and with every instrument module.

\section{RESULTS}

\section{Analytes performance along sigma matrics}

The sigma metrics $(\sigma)$ values of the 20 analytes on HITACHI 7600 - 110 modular biochemistry analyzer, at QC material levels 2 and 3 were calculated based on three (3) TEa sources ( $\sigma_{\text {CLIA, }}$

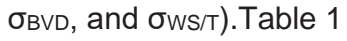

Percentage bias and CV was used on all the $3 \mathrm{TEa}(\mathrm{s})$ to calculate $\sigma$ for all the analytes except with potassium $(\mathrm{K})$ on TEacLIA where absolute bias and standard deviation (SD) was used. Generally $\sigma_{C L I A}>\sigma_{B V D}>\sigma W S / T$ for all the analytes except $\gamma$ GGT, ALT, TBIL, CK, TG, P and Urea whose $\sigma_{B V D}$ was higher than the other two TEa standards. On the other hand, 


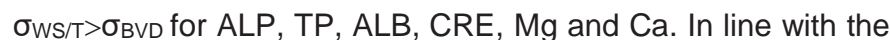
six sigma standard of performance, the analytes were graded into six grades, namely world class $(\sigma \geq 6)$, excellent $(5 \leq \sigma<6)$, good $(4 \leq \sigma<5)$, marginal $(3 \leq \sigma<4)$, poor $(2 \leq \sigma<3)$, and unacceptable $(\sigma<2)$. The $\sigma$ CLIA calculation based on TEacLIA, six analyte; ALP, TBIL, CK, TG, HDL - C, and Mg had a world class performance $(\sigma \geq 6)$, five had excellent performance $(5 \leq$ $\sigma<6$ ); UA, TP (Level 2), CRE, P (Level 3) and K (Level 2). Good $(4 \leq \sigma<5)$ we had five analytes; $\gamma$ - GGT, TP (Level 3), ALB, Ca, and P ( Level 2), three analytes TC (level 2), ALT ( level 3) and $\mathrm{K}$ (Level 3) showed marginal performance $(3 \leq \sigma<4)$. TC (level 3), AST, ALT (level 2), GLU, Urea and Na had poor performance $(2 \leq \sigma<3)$ and no analyte had $\sigma<2$, Tables 1 and 2 .

Table 1: Performance of the 20 analytes on HITACHI 7600 - 110 system and calculated sigma matrics based on different TEa(s).

\begin{tabular}{|c|c|c|c|c|c|c|c|c|c|c|}
\hline & \multicolumn{3}{|c|}{ Average CV (\%) } & \multirow[b]{2}{*}{ TEa } & \multirow[b]{2}{*}{ TEa BVD } & \multirow[b]{2}{*}{ TEa ws/T } & \multicolumn{2}{|l|}{$\sigma_{\text {CLIA }}$} & \multicolumn{2}{|l|}{$\sigma_{\mathrm{BVD}}$} \\
\hline & Bias (\%) & Level 2 & Level 3 & & & & Level 2 & Level 3 & Level 2 & Level 3 \\
\hline g-GGT & 1.38 & 2.91 & 2.73 & 15 & 22.11 & 11 & 4.68 & 4.99 & 7.12 & 7.59 \\
\hline ALP & 2.33 & 3.6 & 3.42 & 30 & 12.04 & 18 & 7.69 & 8.09 & 2.70 & 2.84 \\
\hline UA & 4.95 & 2.29 & 2.09 & 17 & 11.97 & 12 & 5.26 & 5.77 & 3.07 & 3.36 \\
\hline $\mathrm{TC}$ & 2.15 & 2.49 & 2.69 & 10 & 9.01 & 9 & 3.15 & 2.92 & 2.76 & 2.55 \\
\hline AST & 2.62 & 6.71 & 6.89 & 20 & 16.69 & 15 & 2.59 & 2.52 & 2.10 & 2.04 \\
\hline ALT & 3.33 & 5.6 & 5.55 & 20 & 27.48 & 16 & 2.98 & 3.00 & 4.31 & 4.35 \\
\hline GLU & 2.95 & 2.36 & 2.44 & 10 & 6.96 & 7 & 2.99 & 2.89 & 1.70 & 1.64 \\
\hline TP & 1.03 & 1.79 & 1.87 & 10 & 3.63 & 6 & 5.01 & 4.80 & 1.45 & 1.39 \\
\hline TBIL & 0.8 & 2.94 & 2.51 & 20 & 26.94 & 15 & 6.53 & 7.65 & 8.89 & 10.41 \\
\hline ALB & 1.05 & 2.07 & 2.1 & 10 & 4.07 & 6 & 4.32 & 4.26 & 1.46 & 1.44 \\
\hline CRE & 0.91 & 2.53 & 2.73 & 15 & 8.87 & 12 & 5.57 & 5.16 & 3.15 & 2.92 \\
\hline $\mathrm{CK}$ & 1.6 & 2.88 & 2.92 & 30 & 30.3 & 15 & 9.86 & 9.73 & 9.97 & 9.83 \\
\hline $\mathrm{TG}$ & 2.4 & 2.06 & 2.31 & 25 & 25.99 & 15 & 10.97 & 9.78 & 11.45 & 10.21 \\
\hline HDL - C & 8.98 & 1.77 & 1.16 & 30 & 11.63 & & 11.88 & 18.12 & 1.50 & 2.28 \\
\hline $\mathrm{Na}$ & 1.23 & 1.71 & 1.37 & 5 & & 4 & 2.20 & 2.75 & & \\
\hline $\mathrm{Mg}$ & 1.82 & 1.92 & 1.94 & 25 & 4.8 & 15 & 12.07 & 11.95 & 1.55 & 1.54 \\
\hline Ca. & 0.76 & 2.05 & 2.13 & 10 & 2.55 & 5 & 4.51 & 4.34 & 0.87 & 0.84 \\
\hline$P$ & 0.52 & 1.91 & 1.88 & 10 & 10.11 & 10 & 4.96 & 5.04 & 5.02 & 5.10 \\
\hline Urea & 0.82 & 3.69 & 2.92 & 9 & 15.55 & 8 & 2.22 & 2.80 & 3.99 & 5.04 \\
\hline $\mathrm{K}$ & 0.16 & 0.06 & 0.09 & 0.5 & 5.61 & 6 & 5.67 & 3.78 & & \\
\hline
\end{tabular}

CV - Coefficient of variation, TEa - Total allowable error, CLIA - American clinical laboratory improvement amendment 1988, $\sigma$ - Sigma.

BVD - Biological variable data base, WS/T - People's Republic of China Health Industry Standard-WS/T 403-2012.

Table 2: Appropriate QC procedures for the different analytes depending on their sigma performance considering TEaCLIA.

\begin{tabular}{|c|c|c|c|c|}
\hline & $\sigma_{\text {CLIA }}$ & & & \\
\hline & Module & Level2 & Level 3 & QC procedure \\
\hline g-GGT & & 4.68 & 4.99 & $1_{3 \mathrm{~s}} / 2_{2 \mathrm{~s}} / \mathrm{R}_{4 \mathrm{~S}} / 4_{1 \mathrm{~s}}$ with $\mathrm{N} 4$ and R200 \\
\hline ALP & & 7.69 & 8.09 & $1_{3 s} /$ with N2 and R1000 \\
\hline UA & & 5.26 & 5.77 & $1_{3 s} / 2_{2 s} / R_{4 s}$ with $N 2$ and $R 450$ \\
\hline TC & & 3.15 & 2.92 & $1_{3 s} / 2_{2 s} / R_{4 s} / 4_{1 s} / 8_{x}$ with N4 and R45 \\
\hline AST & & 2.59 & 2.52 & $1_{3 s} / 2_{2 s} / R_{4 s} / 4_{1 s} / 8_{x}$ with N4 and R45 \\
\hline ALT & D1 & 2.98 & 3.00 & $1_{3 s} / 2_{2 s} / R_{4 s} / 4_{1 s} / 8_{x}$ with N4 and R45 \\
\hline GLU & & 2.99 & 2.89 & $1_{3 s} / 2_{2 s} / R_{4 s} / 4_{1 s} / 8_{x}$ with N4 and R45 \\
\hline TP & & 5.01 & 4.80 & $1_{3 s} / 2_{2 s} / R_{4 s}$ with N2 and R450 ( level 2), $1_{3 s} / 2_{2 s} / R_{4 s} / 4_{1 s}$ with N4 and R200 ( level 3) \\
\hline ALB & & 4.32 & 4.26 & $1_{3 s} / 2_{2 s} / R_{4 S} / 4_{1 S}$ with $\mathrm{N} 4$ and $\mathrm{R} 200$ \\
\hline TG & & 10.97 & 9.78 & $1_{3 s} /$ with N2 and R1000 \\
\hline HDL - C & & 11.88 & 18.12 & $1_{3 s} /$ with N2 and R1000 \\
\hline Urea & & 2.22 & 2.80 & $1_{3 s} / 2_{2 s} / R_{4 s} / 4_{1 s} / 8_{x}$ with N4 and R45 \\
\hline TBIL & & 6.53 & 7.65 & $1_{3 s} /$ with N2 and R1000 \\
\hline CRE & & 5.57 & 5.16 & $1_{3 s} / 2_{2 s} / R_{4 s}$ with $\mathrm{N} 2$ and $\mathrm{R} 450$ \\
\hline
\end{tabular}




\begin{tabular}{lllll}
\hline CK & P1 & 9.86 & 9.73 & $1_{3 s} /$ with N2 and R1000 \\
Mg & & 12.07 & 11.95 & $1_{3 s} /$ with N2 and R1000 \\
$\mathrm{Ca}$ & & 4.51 & 4.34 & $1_{3 s} / 2_{2 s} / R_{4 s} / 4_{1 s}$ with N4 and R200 \\
$\mathrm{P}$ & & 4.96 & 5.04 & $1_{3 s} / 2_{2 s} / R_{4 s} / 4_{1 s}$ with N4 and R200, (level 2), $1_{3 s} / 2_{2 s} / R_{4 s}$ with N2 and R450 ( Level 3) \\
$\mathrm{Na}$ & ISE & 2.20 & 2.75 & $1_{3 s} / 2_{2 s} / R_{4 s} / 4_{1 s} / 8_{x}$ with N4 and R45 \\
$\mathrm{K}$ & & 5.67 & 3.78 & $1_{3 s} / 2_{2 s} / R_{4 s}$ with N2 and R450 (level 2), $1_{3 s} / 2_{2 s} / R_{4 s} / 4_{1 s} / 8_{x}$ with N4 and R45 (level 3) \\
\hline
\end{tabular}

бCLIA - sigma score of analytes according to CLIA total allowable error standards, D1 - Dispensing module,

P1 - Pippeting module and ISE - Solo ion - selective electrode module.

In addition, based on TEaвvd four analytes, scored world class $(\sigma \geq 6)$, and 10 analytes had $\sigma<3$, For TEaws/t, two analytes; TG (level 2) and Mg had world class score $(\sigma \geq 6)$ and nine analytes scored $\sigma<3$ all shown in table 2 . Three analytes had consistent performance across the three different $\mathrm{TEa}(\mathrm{s})$ (TEacLIA, TEaBVD, and TEaWS/T), TG had a world class sigma score throughout, TBIL scored $\sigma \geq 6$ for TEacLIA and TEaBvd and was excellent under TEaws/T. Likewise, CK scored $\sigma \geq 6$ for TEacLIA and TEabvi and was also good on TEaws/T. However, the following parameters, AST, GLU, Na, and TC scored $\sigma<3$ along all the 3 standards except TC at $\sigma_{C L I A}$ level 2. Figure $1 \& 2$. Likewise three analytes $\mathrm{Ca}$, TP, and ALB had worst sigma performance on TEaBvo standards. Figure $1 \mathrm{C} \& \mathrm{D}$.
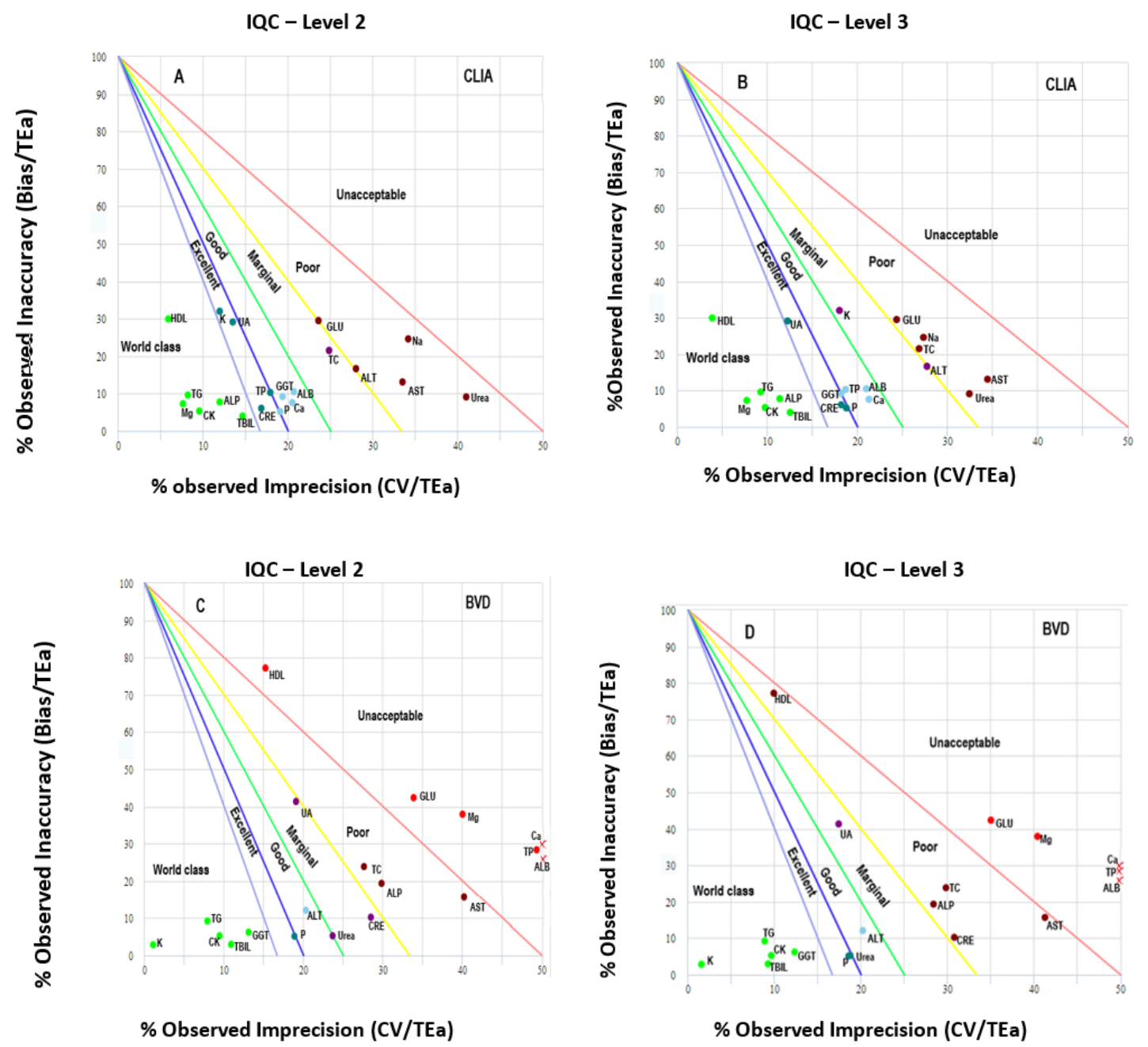

Figure 1: Six sigma performance charts for different analytes; A. Sigma calculated from TEa $a_{C L A}$ and QC material level 2, B. Sigma calculated from TEa $\mathrm{CLIA}_{\mathrm{A}}$ and QC material level 3. C and D, sigma calculated from desirable TEa $\mathrm{BVD}_{\mathrm{D}}$ and QC material levels 2 and 3 respectively. Dots of different colors represent different analytes according to their respective sigma score and stars indicate the worst performing analytes. 

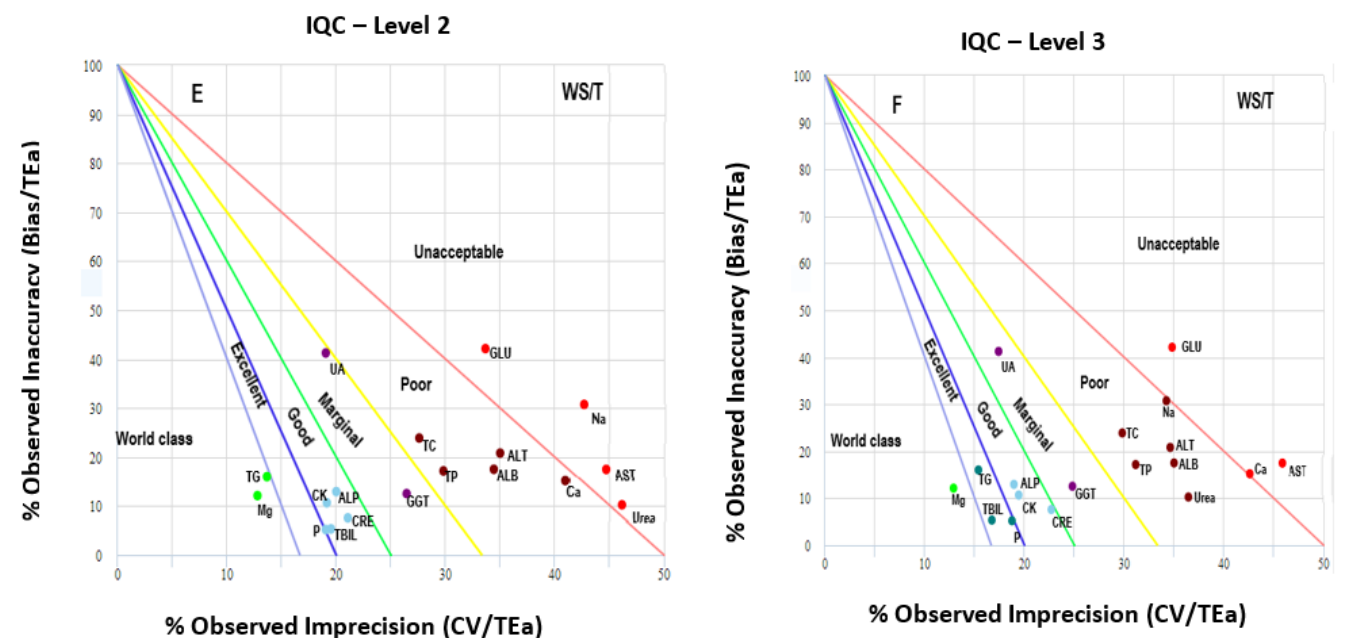

Figure 2: Six sigma performance charts for assays, sigma calculated from TEaws/t at levels 2 and 3 of the QC materials E \& F respectively. Dots of different colors represent different analytes according to their respective sigma score.

All parameters analyzed on P1 module showed world class to good performance with no analyte scoring $\sigma<4$ as compared to other two modules (D1 and ISE) which had a mixture of performance ranging from world class to poor when analyzed on бCLIA, Table 2 and Figure 3.
D1 - Level 2

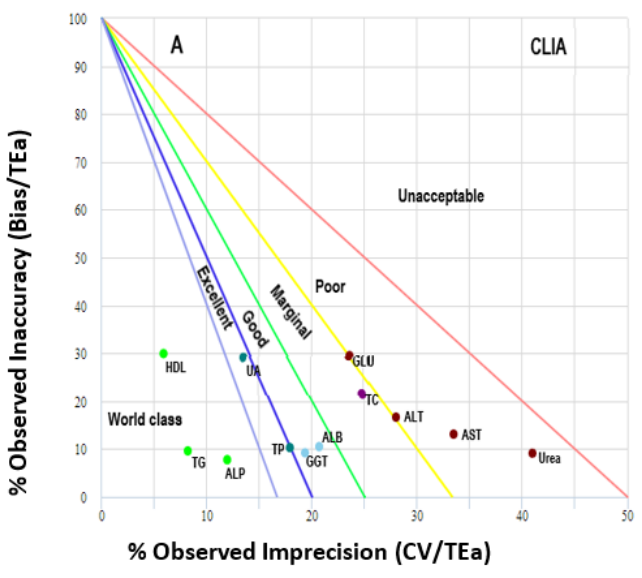

P1 and ISE - Level 2

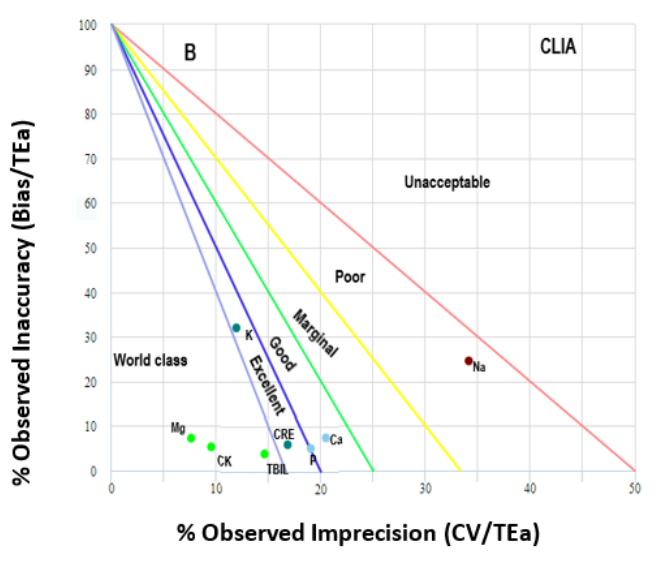

Figure 3: Six sigma performance charts for analyte assays performed by different modules of the analyzer; A. Sigma performance of analytes on D1 module at QC level 2, and TEacLIA, B. Performance of analytes on P1 and ISE modules at QC level 2 and TEacLIA. Dots represent respective sigma scores of the analytes.

\section{Quality improvement QC procedures for different analytes depending on respective sigma performance.}

The hospital clinical biochemical laboratory, currently applies 13s/ N3 (one QC measurement at 3 different QC levels) for the 18 analytes and 13s/ N2 (one QC measurement at 2 different QC levels for $\mathrm{Na}$ and $\mathrm{K}$ ), where " $\mathrm{N}$ " is the number of control measurements per QC event. The redesigned QC procedures for the 20 analytes according to their $\sigma_{C L I A}$ performance at different QC material levels are reported. (Table 2 \& figure 4) For analytes ALP, TG, HDL $-C, T B I L, C K$ and Mg had world class performance $(\sigma \geq 6)$ at both levels of the QC material, a single QC rule (13s), one measurement at two QC material levels (N2) in a single QC event and 1000 (R1000) run size of clinical samples between adjacent QC events. In addition, UA, TP (level 2), CRE, $\mathrm{P}$ (level 3), and $\mathrm{K}$ (level 2) with excellent performance $(5 \leq \sigma<6)$, a multiple sigma rule $13 s / 2_{2 s} / R_{4 s w i t h ~} \mathrm{~N} 2$ and 450 clinical samples between QC events (R450) was adopted.
Analytes, $\gamma$ - GGT, TP (level 3), ALB, Ca, and P (level 2) with good performance $(4 \leq \sigma<5)$ further multiple sigma rules were adopted $1_{3 s} / 2_{2 s} / R_{4 S} / 4_{1 s}$ with N4 and R200. However analytes with marginal, poor and unacceptable performance at both or one of the two levels of QC materials; TC, AST, ALT, GLU, Urea, $\mathrm{Na}$, and $\mathrm{K}$ (level 3 ) with $\sigma<4$, multiple rules were adopted with further reduced clinical sample size between QC runs, $1_{3 S} / 2_{2 S} / R_{4 S} / 4_{1 S} / 8 x$ with N4 and R45.as summarized in table 2. Moreover our results indicate that the sigma metrics analysis could help in redesigning personalized QC procedures for the different analytes as well as at each QC material level unlike the practice of a common sigma rule for all analytes procedure of internal quality control practiced by many clinical biochemistry laboratories. The application of these different rules can enhance a high probability of error detection $\left(\mathrm{P}_{\text {ed }}\right)$ and a low probability of false rejection $\left(P_{\mathrm{fr}}\right)$, a concept of the statistical QC (SQC) procedure based on sigma metrics. 


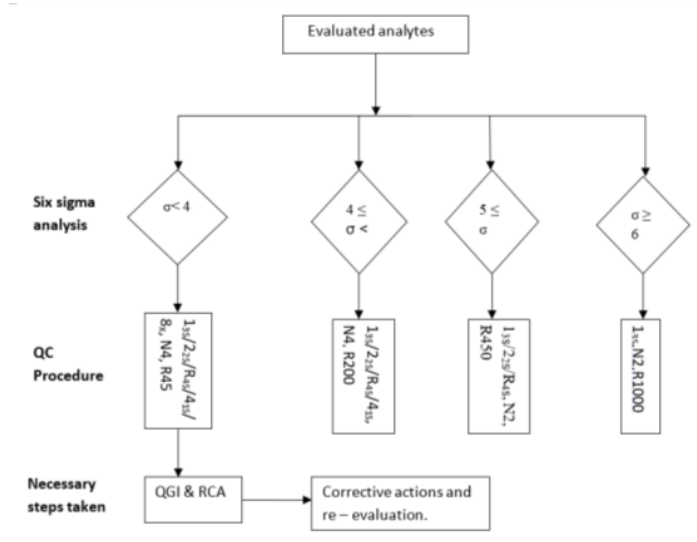

Figure 4: The designed quality improvement strategy for analytes based on sigma matrics and Westgard rules. $\mathrm{N}$ represent number of QC events, R1000 represents run size of patient's samples between QC events similar to R450, R200 and R45.

\section{QGI analysis for the analytes with $\sigma<4$}

QGI analysis was performed to establish the possible causes for the underperforming analyst $(\sigma<4)$. Considering $\sigma_{C L I A}$, five analytes (TC, AST, ALT, Urea, \& Na) showed poor precision at both QC materials, GLU exhibited both imprecision and inaccuracy where as $\mathrm{K}$ showed inaccuracy on QC level 3 materials. (Table 3) In addition, no analyte performed on P1 module had $\sigma<4$. (Table 2) Seven analytes, (TC, AST, TP, ALB, CRE, Mg, \& Ca) exhibited imprecision at both levels of QC materials, Urea was also similar on level 2 QC material. GLU was inaccurate and imprecise on both levels. However, HDL C showed inaccuracy at both $Q C$ levels, putting $\sigma_{B V D}$ into consideration. According to $\sigma \mathrm{WS} / \mathrm{T}$, nine analytes $(\gamma-\mathrm{GGT}, \mathrm{TC}$, AST, ALT, TP, ALB, Ca, \& Na) showed imprecision on both levels of QC material, Urea also had poor precision at QC level 3, UA exhibited inaccuracy at both levels while GLU exhibited both inaccuracy and imprecision. (Table 3)

Table 3: QGI analysis and quality improvement strategies for analytes with $\sigma<4$.

\begin{tabular}{|c|c|c|c|c|c|c|c|c|c|c|c|c|}
\hline & & Averag & CV (\%) & $\sigma_{\text {CLIA }}$ & & $\sigma_{\mathrm{BVD}}$ & & $\sigma_{\mathrm{WS} / \mathrm{T}}$ & & QGI & & \\
\hline & Bias (\%) & Level 2 & Level 3 & Level 2 & Level 3 & Level 2 & Level 3 & Level 2 & Level 3 & Level 2 & Level 3 & Problem \\
\hline g-GGT & 1.38 & 2.91 & 2.73 & $4.68^{a}$ & $4.99^{a}$ & $7.12^{\mathrm{a}}$ & $7.59^{a}$ & 3.31 & 3.52 & 0.3 & 0.3 & Imprecision \\
\hline ALP & 2.33 & 3.6 & 3.42 & $7.69^{a}$ & $8.09^{a}$ & 2.7 & 2.84 & $4.35^{a}$ & $4.58^{\mathrm{a}}$ & 0.4 & 0.5 & Imprecision \\
\hline UA & 4.95 & 2.29 & 2.09 & $5.26^{a}$ & $5.77^{a}$ & 3.07 & 3.36 & 3.08 & 3.37 & 1.4 & 1.6 & Inaccuracy \\
\hline $\mathrm{TC}$ & 2.15 & 2.49 & 2.69 & 3.15 & 2.92 & 2.76 & 2.55 & 2.75 & 2.55 & 0.6 & 0.5 & Imprecision \\
\hline AST & 2.62 & 6.71 & 6.89 & 2.59 & 2.52 & 2.1 & 2.04 & 1.85 & 1.8 & 0.3 & 0.3 & Imprecision \\
\hline ALT & 3.33 & 5.6 & 5.55 & 2.98 & 3.00 & $4.31^{\mathrm{a}}$ & $4.35^{\mathrm{a}}$ & 2.26 & 2.28 & 0.4 & 0.4 & Imprecision \\
\hline GLU & 2.95 & 2.36 & 2.44 & 2.99 & 2.89 & 1.7 & 1.64 & 1.72 & 1.66 & 0.8 & 0.8 & Imprecision \& Inaccuracy \\
\hline TP & 1.03 & 1.79 & 1.87 & $5.01^{\mathrm{a}}$ & $4.80^{\mathrm{a}}$ & 1.45 & 1.39 & 2.78 & 2.66 & 0.4 & 0.4 & Imprecision \\
\hline TBIL & 0.8 & 2.94 & 2.51 & $6.53^{\mathrm{a}}$ & $7.65^{a}$ & $8.89^{a}$ & $10.41^{a}$ & $4.83^{a}$ & $5.66^{a}$ & 0.2 & 0.2 & $\mathrm{~N} / \mathrm{A}$ \\
\hline ALB & 1.05 & 2.07 & 2.1 & $4.32^{\mathrm{a}}$ & $4.26^{\mathrm{a}}$ & 1.46 & 1.44 & 2.39 & 2.36 & 0.3 & 0.3 & Imprecision \\
\hline CRE & 0.91 & 2.53 & 2.73 & $5.57^{a}$ & $5.16^{a}$ & 3.15 & 2.92 & $4.38^{a}$ & $4.06^{a}$ & 0.2 & 0.2 & Imprecision \\
\hline CK & 1.6 & 2.88 & 2.92 & $9.86^{a}$ & $9.73^{a}$ & $9.97^{a}$ & $9.83^{a}$ & $4.65^{\mathrm{a}}$ & $4.59^{a}$ & 0.4 & 0.4 & $\mathrm{~N} / \mathrm{A}$ \\
\hline TG & 2.4 & 2.06 & 2.31 & $10.97^{a}$ & $9.78^{a}$ & $11.45^{\mathrm{a}}$ & $10.21^{a}$ & $6.12^{\mathrm{a}}$ & $5.45^{\mathrm{a}}$ & 0.8 & 0.7 & $\mathrm{~N} / \mathrm{A}$ \\
\hline HDL - C & 8.98 & 1.77 & 1.16 & $11.88^{\mathrm{a}}$ & $18.12^{\mathrm{a}}$ & 1.5 & 2.28 & & & 3.4 & 5.2 & Inaccuracy \\
\hline $\mathrm{Na}$ & 1.23 & 1.71 & 1.37 & 2.2 & 2.75 & & & 1.62 & 2.02 & 0.5 & 0.6 & Imprecision \\
\hline $\mathrm{Mg}$ & 1.82 & 1.92 & 1.94 & $12.07^{\mathrm{a}}$ & $11.95^{\mathrm{a}}$ & 1.55 & 1.54 & $6.86^{\mathrm{a}}$ & $6.79^{a}$ & 0.6 & 0.6 & Imprecision \\
\hline $\mathrm{Ca}$. & 0.76 & 2.05 & 2.13 & $4.51^{\mathrm{a}}$ & $4.34^{\mathrm{a}}$ & 0.87 & 0.84 & 2.07 & 1.99 & 0.2 & 0.2 & Imprecision \\
\hline$P$ & 0.52 & 1.91 & 1.88 & $4.96^{\mathrm{a}}$ & $5.04^{\mathrm{a}}$ & $5.02^{\mathrm{a}}$ & $5.10^{\mathrm{a}}$ & $4.96^{\mathrm{a}}$ & $5.04^{\mathrm{a}}$ & 0.2 & 0.2 & $\mathrm{~N} / \mathrm{A}$ \\
\hline Urea & 0.82 & 3.69 & 2.92 & 2.22 & 2.80 & 3.99 & $5.04^{\mathrm{a}}$ & 1.95 & 2.46 & 0.1 & 0.2 & Imprecision \\
\hline K & 0.16 & 0.06 & 0.09 & $5.67^{\mathrm{a}}$ & 3.78 & & & & & 1.8 & 1.2 & Imprecision \& Inaccuracy \\
\hline
\end{tabular}

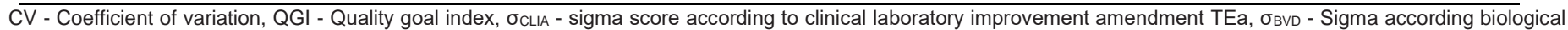

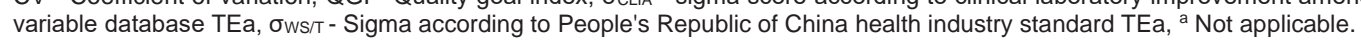

\section{DISCUSSION}

The current study evaluated the performance of 20 analytes on HITACHI 7600 - 110 based on each specific sigma matrics score. Six Sigma techniques uses both bias (system error) and CV (random error) in order to systematically and extensively analyze the system quality standards for proper guidance of clinical laboratories quality management system in identifying the quality improvement strategy. Sigma metrics reveal short comings in precision and accuracy that can be helpful in evaluation of quantitative methods in a clinical laboratory. In addition to bias and CV for computing sigma, total allowable error (TEa) goals from established sources are a requirement. However, TEa, from different sources has a big variation which may contribute to significant differences in the calculated sigma. This study was carried out to evaluate the hospital clinical biochemistry analytical process in terms of quality performance, since quality improvement process starts with problem identification to suggesting possible solutions through systematic root cause analysis (RCA), six sigma is a quantitative method of analysis hence its consideration for significant improvements in laboratory performance is paramount [13]. 
Several studies have been conducted in conjunction with different automatic biochemistry and immunological analyzers considering TEa goals from one or two of CLIA, BVD or WS/T403-2012 but not all at once [14-19]. This study considered TEa goals from three sources, American clinical laboratory amendment 1988, (CLIA 88'), biological variability database,( BVD) and people's republic of China health industry standard, (WS/T403-2012) to evaluate the performance of the three modules, dispensing (D1), pippeting (P1) and solo ion-selective electrode (ISE) of HITACHI 7600 - 110 automatic biochemistry analyzer. To our knowledge this is the first study to evaluate the performance of the three modules of HITACHI $7600-110$ analyzer using six sigma and TEa goals from three different sources (CLIA, BVD, \& WS/T403-2012).

According to our study results, in addition to poor and unacceptable sigma performance by some analytes, three more important findings; first the significant difference in sigma performance of similar analytes across different TEa sources, secondly the sigma performance of analytes performed on different modules of the machine also were quite different with P1 analytes performing better than D1and lastly some analytes showed some inconsistent sigma scores at different levels of the IQC material. The sigma performance was as follows $\sigma_{C L I A}>\sigma_{B V D}>\sigma_{W S} / T$, for most analytes though $\sigma_{B V D}$ had better performance than $\sigma_{C L I A}$ on some analytes. However $\sigma_{W S} / \mathrm{T}$ got the lowest score with majority of analytes scoring $\sigma<4$. This is mainly attributed to the tightness of TEaws/T. The study finding is consistent with some studies like Sigma metrics for assessing the analytical quality of clinical chemistry assays which compared two approaches in calculating sigma [14]. In addition similar inconsistence in sigma scores were observed and reported among sigma performance with different TEa standard sources ${ }^{[15,18]}$. According to the 15 years analytical performance target set in May 2014, by European Federation of Clinical Chemistry and Laboratory Medicine (EFLM) meeting held in Milan, Italy, under the theme "Analytical performance targets", after the Stockholm Conference. Following a detailed discussion by experts in this meeting, on understanding and setting up analytical performance goals in clinical laboratories, issued a supportive statement on the use of TEa values derived from CLIA guidelines [20]. On the other hand, some studies on six sigma performance analysis of clinical biochemistry laboratory have been reported based on TEa from biological variable data base (BVD) $[10,15]$. In support of using TEa from BVD, a Consensus Statement from the 1st Strategic Conference of the European Federation of Clinical Chemistry and Laboratory Medicine, biological variation are model 2 for setting performance specifications ${ }^{[20]}$. In this study we considered the desirable TEaвv due to its suitability given that the optimal TEaBvo is so tight that most analytes will have $\sigma<4$. Furthermore, TEa goals from WS/T 403-2012 are widely applied in China, and several studies on clinical biochemistry laboratory quality performance evaluation have been conducted and reported based on TEaws/T standard [14, 15, 21]. In addition, it's the set standard requirements for use in China, however some analytes like TP and TBIL performance with TEaWS/T was unacceptable because of the tight TEa standards given these parameters had low bias $1.03 \%$ and $0.8 \%$ respectively. Despite the availability of several TEa targets for routine biochemistry, the optimal choice should be established depending on specific laboratory requirements for maintaining high quality standards for intended user satisfaction putting national and international standards into considerations.

The differences in sigma of the same analyte at different levels of QC material is ultimately due to makeable difference in CV\% which is an indicator of poor precision mainly attributed to laboratory personnel and environment. Moreover, this finding is identical to other studies [10,17, 18, 21]. The discrepancy between the QC material levels can partly be attributed to methodology used for some analytes, which might indicate different performance between normal or abnormal concentrations of the QC materials [17]. In line with previous studies, this calls for strict QC procedures in accordance with westgard multiple rules to avoid such discrepancies. Despite the poor and unacceptable sigma performances of some analytes at different TEa standards, their respective Levy Jennings (LJ) charts for both QC materials are with 13 swhich is the QC rule currently applied by our laboratory for all analytes.

The difference in sigma performance exhibited by the different module of the machine was mainly due to the fact that these analytical modules are like different machines. Our results are also no different from one conducted with Beckman Coulter (AU5800) analyzer despite performing similar analytes on different analytical modules of the machine [17].

The incurred variations in sigma values of some analytes in this study as compared to others could be attributed to factors like difference in the methodology, calibrators used, instrument, quality control material used, environmental and personnel factors as well as other pre-analytical factors.

Further, six sigma analysis indicates the quality performance of different analytes or methods in a clinical biochemistry laboratory, though it does not signifying the cause of unwanted performance of the culprit analytes. The current study went ahead to determine QGI for the analytes with $\sigma<4$ for purposes of identifying the problem cause. Majority of the poor to unacceptable analyte performance was due to imprecision (random errors), with only four analytes implicated to inaccuracy (systematic errors).Similar findings are in several previous studies implying most errors encountered in clinical laboratories are human and environmental errors $[10,17,18,21]$. In addition, $\sigma$ values are influenced mainly by two inputs, bias and imprecision, which can be influenced by several factors within a clinical laboratory. In case of unsatisfactory $\sigma$, the cause should be determined, whether it is a bias or imprecision for proper corrective action. The current evaluation study also went ahead to design quality control improvement flow chart to be followed for attainment of the required performance quality. Therefore this study acts as an ice breaker for available opportunities for quality improvement to our clinical biochemistry laboratory which should be implemented and followed by further studies.

\section{CONCLUSION}

Six sigma is a tested, proven excellent tool for statistical quality control (SQC) analysis of the performance of clinical laboratories at different levels, it helps to evaluate the current quality standards of the laboratory, identify excellent performing analytes as well as pointing out those requiring improvement in addition it provides techniques for improvement (Westgard rules). Together with QGI, the possible cause of unwanted analyte performance can be determined. Upon applying it to our hospital clinical biochemistry laboratory, areas of weakness have been identified and quality improvement strategies are already underway and propose a future evaluation study including a comparison in performance between the different modules of the analyzer on similar analytes. However six sigma should be a continuous process and the applied westgard rules should not be permanent but alternated according to analyte performance. In addition, there is need for equipment suppliers, service providers and/or quality control material providers to recommend the most ideal TEa standard source to be applied in evaluation of the specific analyzer, method, and QC reagent 
performance for six sigma analysis.

\section{Author Contributions}

MR and LX; conceived the research idea, formulated the study design, carried out data collection, and initial manuscript write up. NM; contributed in improving the initial manuscript, data analysis and interpretation. All authors read, discussed and approved the final manuscript.

\section{Acknowledgement: None}

\section{Declaration:}

Funding: No funding was received

Conflict of Interest: All authors declare no conflict of interest.

Ethical Approval: Approved by the Institutional Research ethics and Publication Committee.

\section{REFERENCES}

1. Zaini RG, Zaini RG. Physician's satisfaction from laboratory services in maternity and children hospital in Makkah. Int J Lab Med Res. 2015; 1:101.

2. Organization $\mathrm{WH}$. Laboratory quality management system: handbook. World Health Organization, 2011.

3. Allen LC. Role of a quality management system in improving patient safety - Laboratory aspects. Clin Biochem. 2013; 46:1187-93. doi:https://doi.org/10.1016/j.clinbiochem.2013.04.028.

4. Hutchins RJ, Phan KL, Saboor A, Miller JD, Muehlenbachs A. Practical guidance to implementing quality management systems in public health laboratories performing next-generation sequencing: personnel, equipment, and process management (phase 1). J Clin Microbiol. 2019; 57.

5. Kulkarni S, Pierre SA, Kaliaperumal R. Efficacy of Pooled Serum Internal Quality Control in Comparison with Commercial Internal Quality Control in Clinical Biochemistry Laboratory. J Lab Physicians. 2020; 12:191-5.

6. Bayat $H$, Westgard SA, Westgard JO. Planning risk-based statistical quality control strategies: graphical tools to support the new clinical and laboratory standards institute C24-Ed4 guidance. J Appl Lab Med. 2017; 2:211-21.

7. Parvin CA. Planning statistical quality control to minimize patient risk: it's about time. 2018.

8. Westgard JO, Westgard SA. Establishing evidence-based statistical quality control practices. Am J Clin Pathol. 2019; 151:364-70.

9. Westgard JO, Bayat H, Westgard SA. Planning SQC Strategies and Adapting QC Frequency for Patient Risk. Clin Chim Acta. 2021.

10. Goel P, Malik G, Prasad S, Rani I, Manhas S, Goel K. Analysis of performance of clinical biochemistry laboratory using Sigma metrics and Quality Goal Index. Pract Lab Med. 2021; 23:e00195.

11. Emekli DI, Zorbozan N, Erbayraktar Z. Evaluation of Analytical Process Performance by Six Sigma Method. J Basic Clin Heal Sci. 2019; 3:177-83.

12. Westgard S, Bayat H, Westgard JO. Analytical Sigma metrics: A review of Six Sigma implementation tools for medical laboratories. Biochem medica. 2018; 28:174-85.

13. Modi N, Shah T. Application of six sigma test in clinical biochemistry laboratory. Int J Res Med. 2017; 6:75-8.

14. Guo X, Zhang T, Gao X, Li P, You T, Wu Q, et al. Sigma metrics for assessing the analytical quality of clinical chemistry assays: a comparison of two approaches: Electronic supplementary material available online for this article. Biochem medica. 2018; 28:20708.

15. Xia J, Chen S-F, Xu F, Zhou Y-L. Quality specifications of routine clinical chemistry methods based on sigma metrics in performance evaluation. J Clin Lab Anal. 2018; 32.

16. Li R, Wang T, Gong L, Peng P, Yang S, Zhao H, et al. Comparative analysis of calculating sigma metrics by a trueness verification proficiency testing-based approach and an internal quality control data inter-laboratory comparison-based approach. J Clin Lab Anal. 2019; 33:e22989.
17. Zhou B, Wu Y, He H, Li C, Tan L, Cao Y. Practical application of Six Sigma management in analytical biochemistry processes in clinical settings. J Clin Lab Anal. 2020; 34:e23126.

18. Liu Y, Cao Y, Liu X, Wu L, Cai W. Evaluation of the analytical performance of endocrine analytes using sigma metrics. J Clin Lab Anal. 2021; 35:e23581.

19. Cho SE, Nam JW, Hong KS. Performance evaluation of the Hitachi 7600-110 chemistry autoanalyzer. Korean J Clin Pathol. 2001; 21:331-7.

20. Sandberg $S$, Fraser $C G$, Horvath $A R$, Jansen $R$, Jones $G$, Oosterhuis W, et al. Defining analytical performance specifications: Consensus Statement from the 1st Strategic Conference of the European Federation of Clinical Chemistry and Laboratory Medicine. Clin Chem Lab Med. 2015; 53:833-5.

21. Peng S, Zhang J, Zhou W, Mao W, Han Z. Practical application of Westgard Sigma rules with run size in analytical biochemistry processes in clinical settings. J Clin Lab Anal. 2021; 35:e23665. 